\title{
Developing and validating a localised, self-training mindfulness programme for older Singaporean adults: effects on cognitive functioning and implications for healthcare
}

Bryan Wei Hoe $\underline{T a m}^{1}$, Dip, Dana Rui Ting $\underline{L o}^{1}$, Dip, Daniel Wen Hao Seah ${ }^{1}$, Dip, Jun Xian $\underline{L e}^{1}$, Dip, Zann Fang Ying Foo ${ }^{1}$, Dip, Zoe Yu Yah $\underline{\text { Poh }}^{1}$, Dip, Fionna Xiu Jun Thong${ }^{1}$, MA, Sam Kim Yang $\underline{\text { Sim}}^{2}$, MGER, Chew Sim $\underline{\text { Chee }}^{2}$, PhD

\begin{abstract}
There is a paucity of research available on the effect of mindfulness on cognitive function. However, the topic has recently gained more attention due to the ageing population in Singapore, catalysed by recent findings on brain function and cellular ageing. Recognising the potential benefits of practising mindfulness, we aimed to develop a localised, self-training mindfulness programme, guided by expert practitioners and usability testing, for older Singaporean adults. This was followed by a pilot study to examine the potential cognitive benefits and feasibility of this self-training programme for the cognitive function of older adults in Singapore. We found that the results from the pilot study were suggestive but inconclusive, and thus, merit further investigation.
\end{abstract}

Keywords: ageing, cognition, mindfulness, pilot study

\section{INTRODUCTION}

If age-related mental decline could be delayed or prevented, the ramifications for healthcare for the ageing population would be tremendous. The latest research suggests that mindfulness may hold this potential. ${ }^{(1-5)}$ Mindfulness was popularised by Dr Jon Kabat-Zinn from the University of Massachusetts Medical School, Massachusetts, United States, who defined it as the practice of purposefully paying attention in the present moment while being non-judgemental. ${ }^{(6,7)}$ Unlike conventional cognitive training approaches, which require effortful control targeted at training specific brain networks, mindfulness training is general attentional state training, with skills that are transferable to cognition and socio-emotional behaviours. ${ }^{(8)}$ Many studies have supported the beneficial effects of mindfulness-based interventions on health and psychological functioning, including stress, depression and anxiety. ${ }^{(9-15)}$ On the other hand, research on the cognitive effects of mindfulness has only recently begun to draw more attention. This is partly catalysed by increasing population ageing and recent findings on the effects of mindfulness in preserving brain function and cellular ageing. ${ }^{(1,2,16,17)}$

Although research on the cognitive effects of mindfulness is relatively recent and scarce, emerging data has indicated its potential. Studies have found that increased attention, defined as the ability to focus while ignoring other irrelevant information, ${ }^{(18)}$ through the practice of mindfulness was able to enhance working memory. ${ }^{(19,20)}$ This is due to the reciprocal connections between attention and working memory ${ }^{(21)}$ as well as better emotional regulation and the inhibition of secondary elaboration of thoughts. ${ }^{(19,22-25)}$ Working memory is a limited-capacity system, where information is temporarily stored and manipulated. ${ }^{(26)}$ An improvement in sustained attention following mindfulness- based stress reduction was found in some studies, but not in others. ${ }^{(22,27-29)}$ Other studies have also reported the potential effects of mindfulness on inhibitory control (i.e. the ability to override more dominant or automatic responses), cognitive flexibility (the ability to switch between tasks or sets of rules, also referred to as attentional switching) ${ }^{(23-25,27,30-32)}$ and speed of processing (the speed at which a person performs a cognitive activity), ${ }^{(22,33,34)}$ despite other contradictory findings. ${ }^{(35)}$ However, the mixed results could reflect methodological, training or sample differences. ${ }^{(36)}$

In recognition of the potential benefits that mindfulness could bring to the ageing population, we aimed to develop a localised, self-training programme for older Singaporean adults to make mindfulness more widely available to those who would otherwise not be able to access traditional face-to-face classroom training due to various constraints. The localised, self-training programme (in DVD format) was adapted from the standard mindfulness programme to suit older adults by having a shorter training duration and simplified guided instructions (in English and Chinese). For example, Mrazek et al adopted a two-week mindfulness training programme in their study, while Zeidan et al shortened it further to four days. ${ }^{(19,22)}$ Both studies, which reported beneficial effects for cognition (including working memory), were performed on university students, hence limiting its generalisability to older adults. In the current project, material development was guided by input from mindfulness experts and refined after usability tests. The content included body scanning (i.e. guiding one's attentional focus to different parts of the body), mindful breathing, stretching and mindful sitting. ${ }^{(37,38)}$

We herein describe our pilot study that examined the efficacy of the self-training mindfulness programme specifically for cognitive function in older adults. This study was conceived in cognisance

${ }^{1}$ Psychology Studies, School of Humanities \& Social Sciences, ${ }^{2}$ Centre for Ageing Studies, Temasek Polytechnic, Singapore

Correspondence: Dr Chee Chew Sim, Researcher, Centre for Ageing Studies, Temasek Polytechnic, 21 Tampines Avenue 1, Singapore 529757. ccsim@tp.edu.sg 
of the limited research on mindfulness, including: (a) the effects of mindfulness on cognition; (b) the feasibility of a self-training mindfulness programme; (c) the viability of a shorter training duration; (d) its applicability to local context; and (e) the ageing population. To the best of our knowledge, this pilot study is one of the first to investigate a self-training mindfulness programme.

\section{METHODS}

We recruited 30 participants (English- or Mandarin-speaking) who were aged $\geq 55$ years. A total of 23 participants who met the eligibility criteria (Mini-Mental State Examination score $>25$ and Geriatric Depression Scale score $<5$ ) were eventually included in the study. 13 of the participants were from a senior activity centre and ten were from a database of research participants. Participants were assigned to the experimental group or active control group based on a randomised block design using an online research randomiser. Before testing, participants' baseline performance for the cognitive outcome measures (Table I) was assessed.

During the two-week intervention, the experimental group practised mindfulness exercises following the guided DVD programme for about 20 minutes per day, five days per week, while the active control group watched an educational video series on the history of kungfu. Participants from the Centre for Ageing Studies database did the training at home using the DVD, with a training log to monitor their training compliance. The participants recruited from the senior activity centre, who had healthy function, did the same training at the centre following the assigned DVD programme, with experimenters present to set up the audio-visual equipment, record training attendance and provide minimal prompts. After testing, participants underwent the same cognitive assessments.

\section{RESULTS AND DISCUSSION}

At baseline, there were no significant differences between the experimental and active control groups for both demographic variables (years of education: experimental $5.00 \pm 4.00 \mathrm{vs}$. control $7.18 \pm 5.60, p=0.29$; and age: experimental $72.92 \pm 9.37$ vs. control $72.45 \pm 9.32, p=0.91)$ and cognitive performance (Table II).

Taken together, there was an overall improvement in the mindfulness and executive functions of the participants. Nonetheless, the improvement in the training group was not significantly greater than in the control group $(p=0.34)$. In order to conduct a manipulation check, we included the Mindful Attention Awareness Scale to measure the level of mindfulness of participants before and after training; the results suggested a general improvement. Although the training group improved as hypothesised following the mindfulness programme, the improvement was not significantly greater than that of the active control group. This could be due to the limitations of the self-report method, which a significant proportion of participants had some difficulty understanding. Investigators had to explain the method to these participants in their own dialects. This is a recognised challenge for the current cohort of older adults in Singapore who are illiterate or not highly educated. It is also probable that

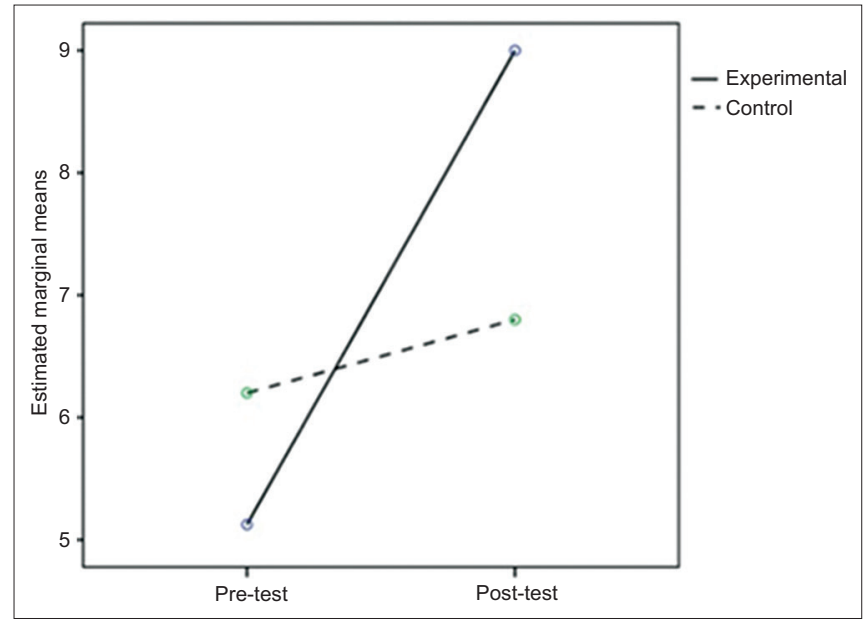

Fig. 1 Performance results of story recall (episodic memory) for centrebased participants as a function of training groups $(n=13)$, controlling for education and age.

Table I. Summary of outcome measures.

\begin{tabular}{ll}
\hline Cognitive function & Measure \\
\hline Mindful attention & Mindful Attention Awareness Scale \\
Working memory & Digit span \\
Episodic memory & Story recall \\
Executive functions & Stroop effect \\
& Trail making test (part B) \\
& Design fluency \\
& Verbal fluency \\
& Trail making test (part A) \\
Speed of processing & Symbol search \\
& Symbol digit modalities test \\
\hline
\end{tabular}

the study was not sufficiently powered. Nonetheless, the overall improvement in mindfulness was in line with the aim of the training.

Furthermore, this study found that the effects of the localised, self-training mindfulness programme differentially impacted participants depending on the training mode. Notably, centrebased participants in the mindfulness group exhibited significant improvement $(p=0.007)$ in episodic memory as compared to the active control group ( $p=0.501$ ) (Fig. 1). It was also noted that home-based participants with a higher educational level generally outperformed centre-based participants. Thus, the margin to capture improvement could be greater for centre-based participants (i.e. they started at a lower baseline performance), which reflects that those with a greater range for improvement would benefit most from this programme.

\section{CONCLUSION}

Mindfulness is recognised as a promising, low-cost and noninvasive intervention that can potentially buffer against cognitive decline or impairment. ${ }^{(3-5)}$ This pilot study has partially shown that short-term gains from mindfulness training are possible, which is consistent with studies from Mrazek et al and Zeidan et al. ${ }^{(19,22)}$ Nonetheless, future studies are needed to determine the long-term impact of consistent mindfulness training over a longer period of time. More empirical evidence is needed from the nascent but 
Table II. Outcome measures before and after testing ( $n=23)$.

\begin{tabular}{|c|c|c|c|c|}
\hline \multirow[t]{2}{*}{ Measure } & \multicolumn{2}{|c|}{ Experimental group $(n=12)$} & \multicolumn{2}{|c|}{ Active control group $(n=11)$} \\
\hline & Before & After & Before & After \\
\hline Mindful Attention Awareness Scale (mean rating) & $2.64 \pm 0.33$ & $2.88 \pm 0.36$ & $2.81 \pm 0.56$ & $2.88 \pm 0.58$ \\
\hline Digit span (no. of correct sequences recalled) & $5.04 \pm 0.81$ & $5.14 \pm 0.87$ & $4.86 \pm 1.05$ & $5.32 \pm 0.78$ \\
\hline Story recall (no. of correct story units recalled) & $7.75 \pm 4.83$ & $10.58 \pm 4.34$ & $8.73 \pm 4.38$ & $10.36 \pm 5.47$ \\
\hline Stroop effect (interference T-score) & $50.33 \pm 8.70$ & $51.58 \pm 11.41$ & $48.18 \pm 11.21$ & $49.91 \pm 8.19$ \\
\hline Trail making test (part B) (1/time taken $[\mathrm{s}])$ & $0.0102 \pm 0.0080$ & $0.0113 \pm 0.0062$ & $0.0103 \pm 0.0036$ & $0.0114 \pm 0.0059$ \\
\hline Design fluency (no. of correct responses) & $20.92 \pm 8.20$ & $23.00 \pm 8.22$ & $19.40 \pm 7.18$ & $22.00 \pm 8.82$ \\
\hline Verbal fluency (no. of correct responses) & $39.91 \pm 5.67$ & $41.45 \pm 5.13$ & $40.09 \pm 5.67$ & $39.00 \pm 7.01$ \\
\hline Trail making test (part A) (1/time taken $[s])$ & $0.0218 \pm 0.0096$ & $0.0219 \pm 0.0069$ & $0.0208 \pm 0.0095$ & $0.0236 \pm 0.0072$ \\
\hline $\begin{array}{l}\text { Symbol search (no. of correct responses adjusted for } \\
\text { errors) }\end{array}$ & $18.91 \pm 6.50$ & $18.45 \pm 6.86$ & $16.64 \pm 8.60$ & $18.40 \pm 9.70$ \\
\hline Symbol digit modalities test (no. of correct responses) & $74.42 \pm 28.90$ & $75.17 \pm 24.41$ & $70.73 \pm 29.22$ & $75.36 \pm 27.97$ \\
\hline
\end{tabular}

Data presented as mean \pm standard deviation.

burgeoning research on the effects of mindfulness on cognition. The present study is an attempt to answer this need. It makes an incremental contribution to the field as a pilot study to show the feasibility and potential cognitive benefits of a self-training mindfulness programme that merits further investigation on a larger scale. Future research can explore other training formats, including mobile applications, to propagate mindfulness training.

\section{ACKNOWLEDGEMENTS}

This study was supported by internal funding from Temasek Polytechnic, Singapore. We thank Chan Ying Ying, Lim Ayaka, Muhhamad Syafiq Md Yusof, Poh Wei Jun and Saloni Shirish Balekundri for the development of the training material. We are grateful to Dr Ong Seh Hong, Eelin Ong and Dr Phang Cheng Kar for their valuable advice in developing the materials; AWWA Senior Activity Centre for support in recruitment and the training facility; Wei Kong, Chua Ping Tzeun, and Dr Tan Wah Pheow for their advice in project planning; and more importantly, all the research participants who made this study possible.

\section{REFERENCES}

. Hölzel B, Carmody J, Vangel M, et al. Mindfulness practice leads to increases in regional brain gray matter density. Psychiatry Res 2011; 191:36-43.

2. Jacobs TL, Epel ES, Lin J, et al. Intensive meditation training, immune cell telomerase activity, and psychological mediators. Psychoneuroendocrinology $2011 ; 36: 664-81$.

3. Segall S, Black DS. Can Meditation Slow the Aging Process? In: American Society on Aging [online]. Available at: http://www.asaging.org/blog/can-meditationslow-aging-process. Accessed January 17, 2015.

4. Xiong GL, Doraiswamy PM. Does meditation enhance cognition and brain plasticity? Ann N Y Acad Sci 2009; 1172:63-9.

5. Larouche E, Hudon C, Goulet S. Potential benefits of mindfulness-based interventions in mild cognitive impairment and Alzheimer's disease: an interdisciplinary perspective. Behav Brain Res 2015; 276:199-212.

6. Kabat-Zinn J. Full catastrophe living: using the wisdom of your body and mind to face stress, pain, and illness. New York: Delacorte Press, 1990.

7. Kabat-Zinn J. Wherever You Go, There You Are: Mindfulness Meditation In Everyday Life. London: Piatkus Books, 2001.

8. Tang YY, Posner MI. Attention training and attention state training. Trends Cogn Sci 2009; 13:222-7.

9. Eberth J, Sedlmeier P. The effects of mindfulness meditation: a meta-analysis. Mindfulness 2012; 3:174-89.

10. Khoury B, Lecomte T, Fortin G, et al. Mindfulness-based therapy: a comprehensive meta-analysis. Clin Psychol Rev 2013; 33:763-71.

11. Baer RA. Mindfulness Training as a Clinical Intervention: A Conceptual and Empirical Review. Clin Psychol Sci Pract 2003; 10:125-43.

12. Hollon SD, Ponniah K. A review of empirically supported psychological therapies for mood disorders in adults. Depress Anxiety 2010; 27:891-932.

13. Evans S, Ferrando S, Findler M, et al. Mindfulness-based cognitive therapy for generalized anxiety disorder. J Anxiety Disord 2008; 22:716-21.
14. Nyklícek I, Kuijpers K. Effects of mindfulness-based stress reduction intervention on psychological well-being and quality of life: is increased mindfulness indeed the mechanism? Ann Behav Med 2008; 35:331-40.

15. Kaviani H, Javaheri F, Hatami N. Mindfulness-based cognitive therapy (MBCT) reduces depression and anxiety induced by real stressful setting in non-clinical population. Int J Psychol Psychol Ther 2011; 11:285-96.

16. Chiesa A, Serretti A. A systematic review of neurobiological and clinical features of mindfulness meditations. Psychol Med 2011; 40:1239-52.

17. Desbordes G, Negi LT, Pace TW, et al. Effects of mindful-attention and compassion meditation training on amygdala response to emotional stimuli in an ordinary, non-meditative state. Front Hum Neurosci 2012; 6:292.

18. Goldstein EB. Cognitive Psychology: Connecting Mind, Research and Everyday Experience. 3rd ed. Belmont, CA: Wadsworth, Cengage Learning, 2011

19. Mrazek MD, Franklin MS, Phillips DT, Baird B, Schooler JW. Mindfulness training improves working memory capacity and GRE performance while reducing mind wandering. Psychol Sci 2013; 24:776-81.

20. Jensen CG, Vangkilde S, Frokjaer V, Hasselbalch SG. Mindfulness training affects attention--or is it attentional effort? J Exp Psychol Gen 2012; 141:106-23.

21. Awh E, Vogel EK. The bouncer in the brain. Nat Neurosci 2008; 11:5-6.

22. Zeidan F, Johnson SK, Diamond BJ, David Z, Goolkasian P. Mindfulness meditation improves cognition: evidence of brief mental training. Conscious Cogn 2010; 19:597-605.

23. Ortner CN, Kilner SJ, Zelazo PD. Mindfulness meditation and reduced emotional interference on a cognitive task. Motiv Emotion 2007; 31:271-83.

24. Allen M, Dietz M, Blair KS, et al. Cognitive-affective neural plasticity following active-controlled mindfulness intervention. J Neurosci 2012; 32:15601-10.

25. Prakash RS, Hussain MA, Schirda B. The role of emotion regulation and cognitive control in the association between mindfulness disposition and stress. Psychol Aging 2015; 30:160-71.

26. Baddeley A. The episodic buffer: a new component of working memory? Trends Cogn Sci 2000; 4:417-423.

27. Chambers R, Lo BC, Allen NB. The impact of intensive mindfulness training on attentional control, cognitive style, and affect. Cogn Ther Res 2008; 32:303-22.

28. Cusens B, Duggan GB, Thorne K, Burch V. Evaluation of the breathworks mindfulness-based pain management programme: effects on well-being and multiple measures of mindfulness. Clin Psychol Psychother 2010; 17:63-78.

29. MacCoon DG, MacLean KA, Davidson RJ, Saron CD, Lutz A. No sustained attention differences in a longitudinal randomized trial comparing mindfulnessbased stress reduction versus active control. PLoS One 2014; 9:e97551.

30. Bjorklund DF. Children's Thinking: Cognitive Development. 5th ed. Belmont, CA: Wadsworth, 2012

31. Shapiro SL, Carlson LE, Astin JA, Freedman B. Mechanisms of mindfulness. J Clin Psychol 2006; 62:373-86.

32. Bishop SR, Lau M, Shapiro S, et al. Mindfulness: a proposed operational definition. Clin Psychol Sci Pract 2004; 11:230-41.

33. Salthouse TA. The processing-speed theory of adult age differences in cognition. Psychol Rev 1996; 103:403-28.

34. Johansson B, Bjuhr H, Rönnbäck L. Mindfulness-based stress reduction (MBSR) improves long-term mental fatigue after stroke or traumatic brain injury. Brain Inj 2012; 26:1621-8.

35. Josefsson T, Lindwall $M$, Broberg AG. The effects of a short-term mindfulness based intervention on self-reported mindfulness, decentering, executive attention, psychological health, and coping style: examining unique mindfulness effects and mediators. Mindfulness 2014; 5:18-35.

36. Chiesa A, Calati R, Serretti A. Does mindfulness training improve cognitive abilities? A systematic review of neuropsychological findings. Clin Psychol Rev $2011 ; 31: 449-64$

37. Stahl B, Goldstein E. A mindfulness-based stress reduction workbook. Oakland, CA: New Harbinger Publications, 2010.

38. Williams M, Teasdale J, Segal Z, Kabat-Zinn J. The Mindful Way Through Depression: Freeing Yourself from Chronic Unhappiness. New York: The Guilford Press, 2007. 